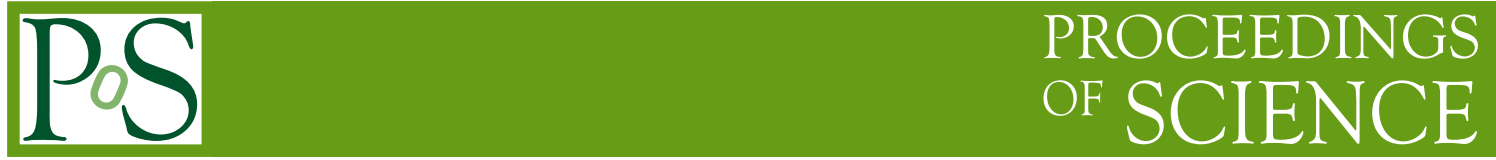

\title{
Thermodynamics using p4-improved staggered fermion action on QCDOC
}

\section{Chulwoo Jung* for the RBC-Bielefeld Collaboration}

Brookhaven National Laboratory and Columbia University, USA

E-mail: chulwoo@bnl.gov

We present an exploratory study of the thermodynamics of $N_{f}=3$ QCD with an improved staggered fermions using the QCDOC supercomputer. We use a p4 action with MILC-style smeared links (Fat 7). Some details of the implementation of the 44 action on QCDOC are discussed and performance benchmarks are given. We show preliminary results for the quark mass dependence of the pseudo-critical temperature $T_{c}$ from several lattice volumes. We also make a comparison between $\mathrm{p} 4 \mathrm{fat} 7$ and the old $\mathrm{p} 4$ action.

XXIIIrd International Symposium on Lattice Field Theory

25-30 July 2005

Trinity College, Dublin, Ireland

\footnotetext{
${ }^{*}$ Speaker.
} 


\section{Introduction}

Lattice QCD is an important tool for the study of finite temperature QCD. Typically one is forced to use larger lattice spacing $(a)$ for finite temperature quantities than zero temperature quantities. This is mainly because the temperature regime of interest $T_{c}=\left(N_{\tau} a\right)^{-1}\left(N_{\tau}=4-8\right)$ is about (1 $\mathrm{fm})^{-1}$, and simulations at several gauge couplings have to be performed to locate the transition temperature.

This makes improved staggered fermion formulations attractive for thermodynamics studies. Improvements of rotational symmetry via "knight's term" (p4 action) largely reduces the cut-off effects in the pressure and energy density at high temperatures [1]. Also, it is well known that staggered fermions break the flavor symmetry of continuum QCD and this effect can be largely reduced if fat links are used in the fermion action [2]. In this contribution we present an exploratory study of QCD phase diagram with a fermion action which combines the improvements in [1] and [2](p4fat7 action). A more detailed description of the action and simulation parameters are given in section 2. The phase transition for 3 degenerate flavors of p4fat7 quark is described in section 3 . Comparison between p4fat7 and p4 action used in [3] is described in section 4.

\section{Action and simulation parameters}

For this study we use the tree-level improved Symanzik gauge action and p4fat7 action.

$$
\begin{gathered}
S(x)=\beta S_{g}(x)+S_{f}(x), \\
S_{g}(x)=\sum_{\mu>v}\left[\frac{5}{3}\left(1-\frac{1}{3} \operatorname{Re} \operatorname{Tr}\left[U_{\mu}(x) U_{v}(x+\hat{\mu}) U_{\mu}^{\dagger}(x+\hat{v}) U_{v}^{\dagger}(x)\right]\right)\right. \\
\left.-\frac{1}{6}\left(1-\frac{1}{6} \operatorname{Re} \operatorname{Tr}\left[U_{\mu}(x) U_{\mu}(x+\hat{\mu}) U_{v}(x+2 \hat{\mu}) U_{\mu}^{\dagger}(x+\hat{\mu}+\hat{v}) U_{\mu}^{\dagger}(x+\hat{v}) U_{v}^{\dagger}(x)\right]-\frac{1}{6} \operatorname{Re} \operatorname{Tr}[\mu \leftrightarrow v]\right)\right] .
\end{gathered}
$$

The fermion part of the action $\left(S_{f}\right)$ is shown in Fig. 1. The coefficient in the smeared link are chosen to cancel flavor symmetry breaking at order $\alpha_{s} a^{2}$ [2],

$$
c_{1}=\frac{1}{8}, \quad c_{3}=\frac{1}{16}, \quad c_{5}=\frac{1}{64}, \quad c_{7}=\frac{1}{384} .
$$

Here we use the above values of the fat7 coefficients without tadpole improvement. The effect of tadpole improvement on quenched configurations is studied in [4].

All the codes needed for dynamical gauge evolution (HMD R algorithm) is implemented for QCDOC in Columbia Physics System (CPS) and numerically checked against existing Asqtad and p4 action results. The "knight's move" term of $\mathrm{p} 4$ action is implemented without non-nearest communication by breaking the term into Parallel transport $\left(\psi_{\mu}^{\prime}(x)=U_{\mu}(x) \psi(x+\hat{\mu})\right)$ and recombination $\left(R_{\mu}(x)=\sum_{v \neq \mu}(\psi(x+\hat{v}) \pm \psi(x-\hat{v}))\right.$, as illustrated in Fig. 2.

Inversion of p4fat7 Dirac operator, which is the dominating part of gauge evolution for small masses, is currently running at $\sim 31 \%$ of the peak for $4^{3} \times 6$ local volume, which is the local 

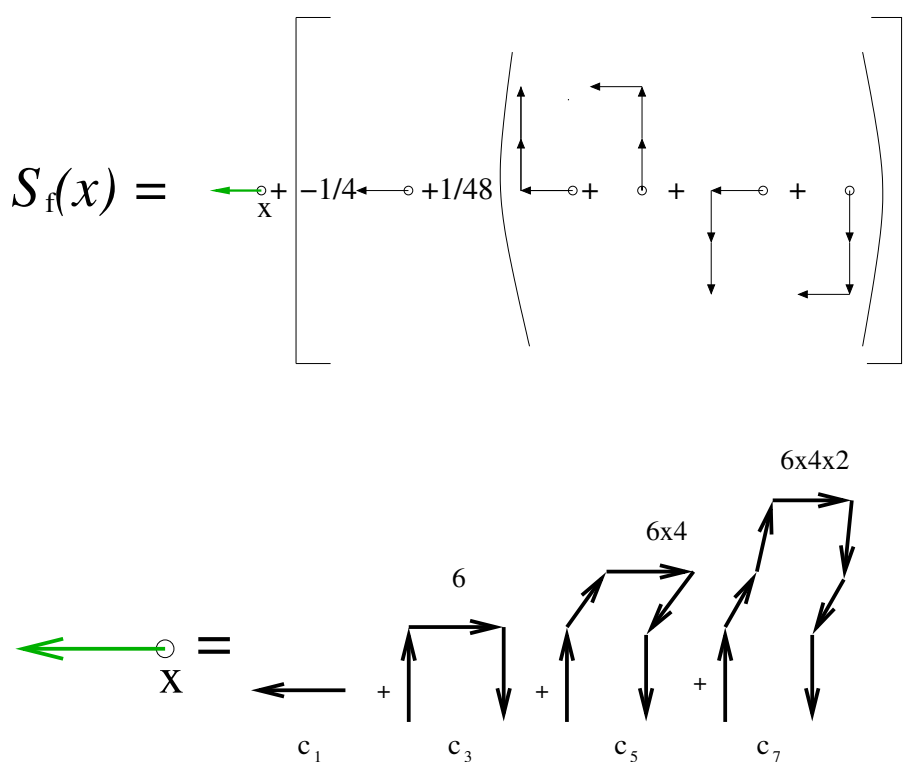

Figure 1: Diagram of p4fat7 action. The numbers above graphs denote the multiplicities of each graphs.

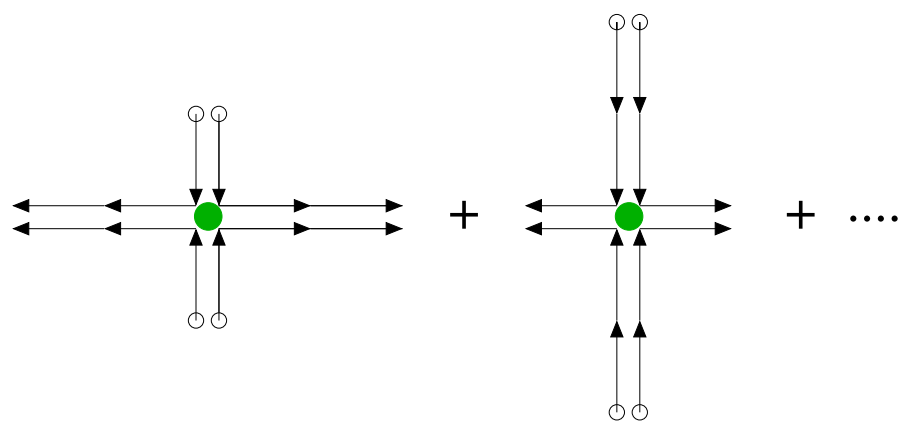

Figure 2: Illustration of implementation of $\mathrm{p} 4$ action. The arrows and circles denote the parallel transports and recombinations respectively.

volume for $32^{3} \times 6$ on a 512-node QCDOC partition. Further optimization of the Dirac operator, especially at smaller volumes, is possible. Rational Hybrid Monte Carlo(RHMC)[5] implementation is also in progress. All the simulations were done with $\mathrm{R}$ algorithm[6] with the step size equal to $40 \%$ of the bare mass in lattice units.

\section{3. $N_{f}=3$ phase transition with p4fat7}

Fig. 3 shows the coupling at the transition as a function of quark masses in lattice units. $\beta_{c}$ are located by measuring susceptibilities in average plaquette, Polyakov loop and chiral condensate. The location of peaks coincide within errors. The use of tree-level coefficients enables the use of the reweighting procedure, which is used in locating the transition temperature. At $\beta_{c}$ for each mass, separate measurements of heavy quark potential and spectrum calculations were done on $16^{3} \times 32$ lattices for physical scale setting. 


\begin{tabular}{l|l|l|l}
\hline Volume & sites/node & CG(MFlops/node) & Smearing(MFlops/node) \\
\hline $2^{2} 4^{2}$ & 64 & 173 & 271 \\
$4^{4}$ & 256 & 207 & 313 \\
$4^{2} \times 6^{2}$ & 576 & 264 & 264 \\
$6^{4}$ & 1296 & 231 & 164 \\
\hline
\end{tabular}

Table 1: Performance of p4fat7 evolution codes on QCDOC, 420Mhz, 1024 nodes, MFlops/node
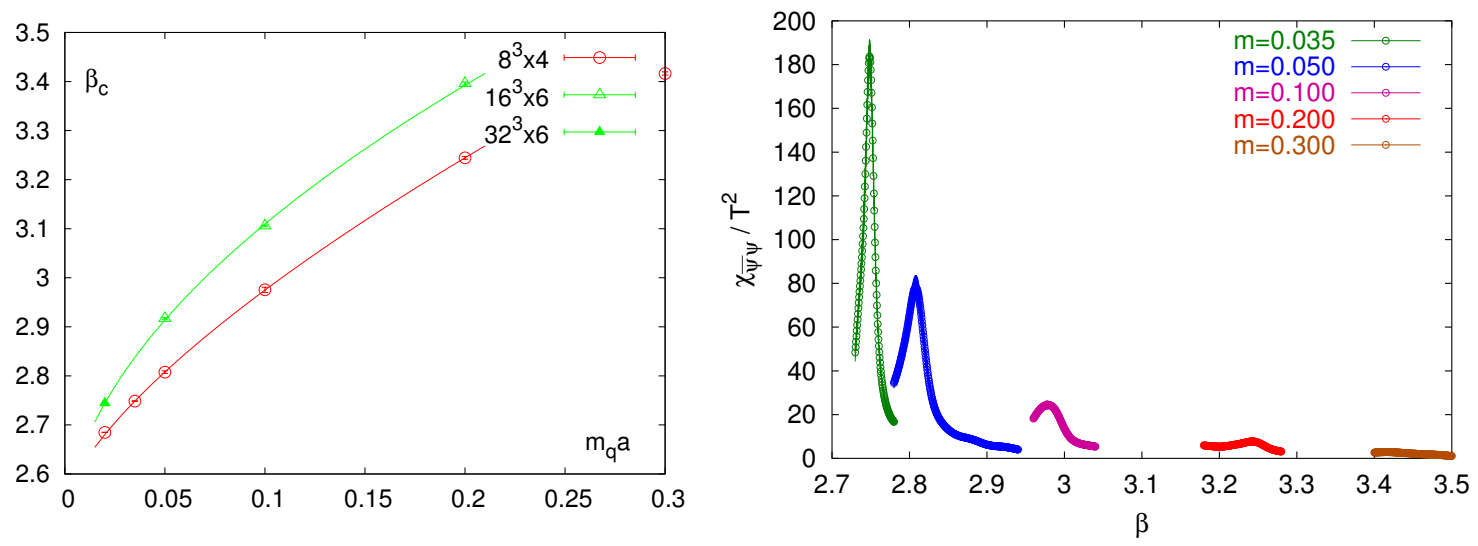

Figure 3: Transition coupling in lattice units $\left(\beta_{c}\right)$ for 3 flavor $\mathrm{p} 4$ fat 7 action. The curves represent fits to $\beta_{c}\left(m_{q}\right)=A+B\left(m_{q} a\right)^{C}$. Red and green points denote $N_{\tau}=4$ and $N_{\tau}=6$ respec-

Figure 4: The susceptibility in chiral condensate for $8^{3} \times 4$ tively.

It has been shown that staggered type fermions exhibits a transition from a rapid crossover to a 1st order transition in small masses. Initial studies suggest that the mass where the transition occurs for $N_{\tau}=4$ may be larger than [3], as shown by the rapidly increasing peak in susceptibility in Fig. 4. This may indicate the additional smearing does not suppress finite lattice spacing error much. Further study is in progress.

Fig. 5 shows the heavy quark potential and the lattice spacing, measured by $r_{0}$ [7]. The error in each $T_{c} r_{0}$ includes the difference between 2 different spacial smearings.

\section{Comparison with 4 fat 3 action}

P4fat3 action, used in [3], has the knight's move term, 1-link term as well as 3-staple terms. In the notation in Fig. 1, the coefficients are

$$
c_{1}=\frac{3}{4} \frac{1}{1+6 \omega}, \quad c_{3}=\frac{3}{4} \frac{\omega}{1+6 \omega}, \quad c_{5}=c_{7}=0, \quad \omega=0.2 .
$$



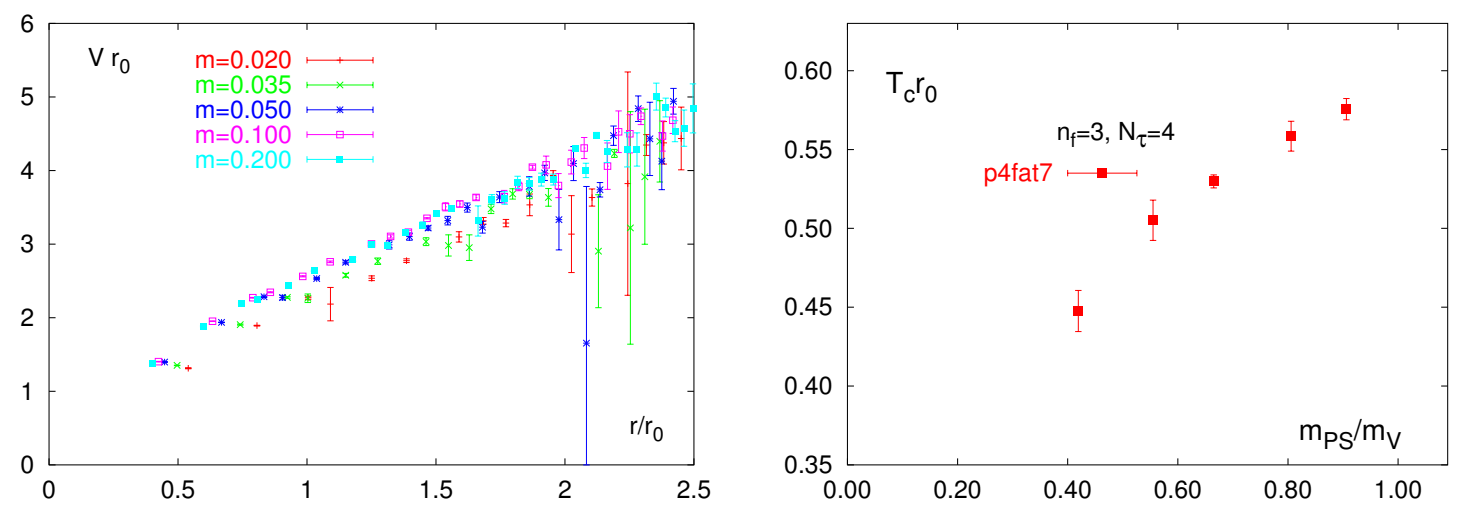

Figure 5: Scale from heavy quark potential for $8^{3} \times 4$ p4fat7 lattices

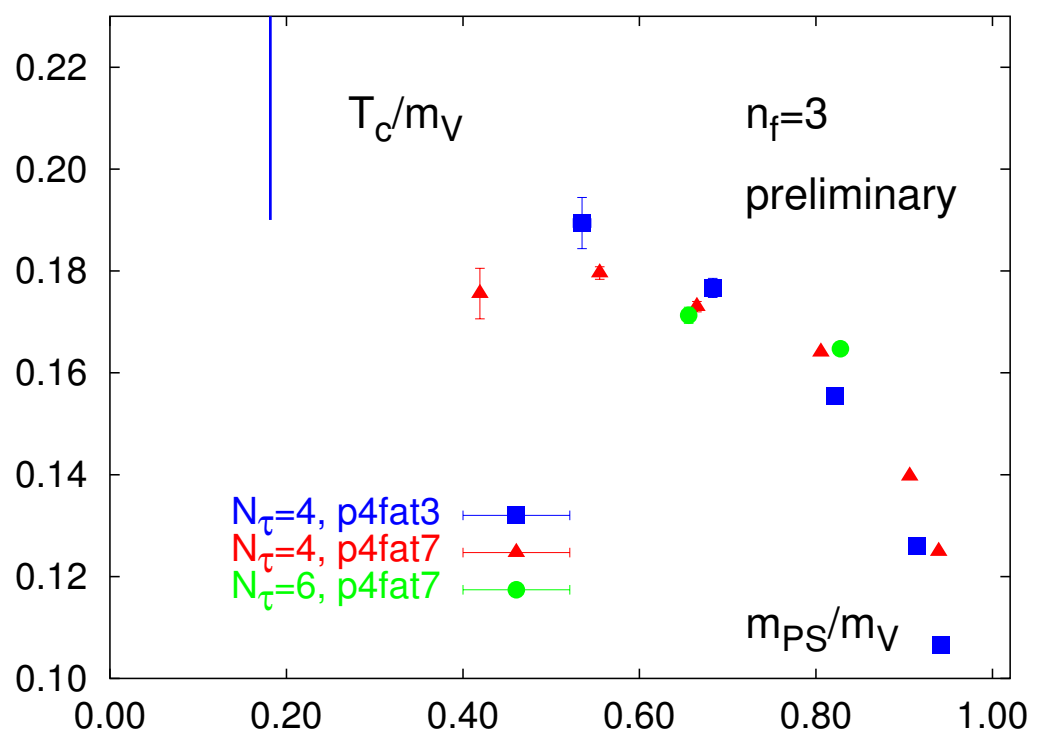

Figure 6: Comparison of $T_{c} / m_{V}$ between $\mathrm{p} 4 \mathrm{fat} 7$ and the $\mathrm{p} 4 \mathrm{fat} 3$.

Fig. 6 shows the behavior of the transition temperature for the p4fat7 and p4fat 3 actions. The transition temperature in units of vector meson mass shows a good agreement between the 2 actions. A further study will be needed for the continuum extrapolation.

\section{Summary and Future plans}

We studied the phase transition of p4fat7 action for 3 degenerate flavors of quarks. p4fat7 action exhibits the finite temperature phase transition and appears to be suitable for other studies such as Equation of States. Although the use of fat7 smearing shows the expected reduction of flavor symmetry breaking symmetry breaking in smaller lattice spacings[4], we do not see a large improvement in flavor symmetry breaking for the lattice spacing relevant for $N_{\tau}=4$. More improvement 
such as employing tadpole improvement may be needed. We also showed some preliminary results for $N_{\tau}=6$ phase transition. More systematic study of $N_{f}=3$ and $2+1$ transition is in progress. Dirac operator and other routines needed for $\mathrm{p} 4 \mathrm{fat} 7 \mathrm{Hybrid}$ Moleculardynamics are optimized for QCDOC and performing at $\sim 30 \%$ for the volumes relevant for finite temperature studies. Implementation for RHMC is under way.

\section{References}

[1] U. M. Heller, F. Karsch and B. Sturm, Improved staggered fermion actions for qcd thermodynamics, Phys. Rev. D60 (1999) 114502 [hep-lat/9901010].

[2] MILC Collaboration, K. Orginos, D. Toussaint and R. L. Sugar, Variants of fattening and flavor symmetry restoration, Phys. Rev. D60 (1999) 054503 [hep-lat/ 9903032$].$

[3] F. Karsch, E. Laermann and A. Peikert, The pressure in 2, 2+1 and 3 flavour qcd, Phys. Lett. B478 (2000) 447-455 [hep-lat/ 0002003 ].

[4] M. Cheng, Scaling test of the p4-improved staggered fermion action, in XXIIIrd International Symposium on Lattice Field Theory, Pos(LAT2005)150.

[5] M. A. Clark and A. D. Kennedy, The rhmc algorithm for 2 flavors of dynamical staggered fermions, Nucl. Phys. Proc. Suppl. 129 (2004) 850-852 [hep-lat/ 0309084 ].

[6] S. A. Gottlieb, W. Liu, D. Toussaint, R. L. Renken and R. L. Sugar, Hybrid molecular dynamics algorithms for the numerical simulation of quantum chromodynamics, Phys. Rev. D35 (1987) 2531-2542.

[7] ALPHA Collaboration, M. Guagnelli, R. Sommer and H. Wittig, Precision computation of a low-energy reference scale in quenched lattice qcd, Nucl. Phys. B535 (1998) 389-402 [hep-lat/9806005].

\section{Acknowledgments}

We thank Frithjof Karsch, Peter Petreczky, Konstantin Petrov, Christian Schmidt, Norman Christ, Robert Mawhinney and Michael Cheng for the software developments and data analysis for this proceeding, as well as useful and enlightening discussions.

We also thank Peter Boyle, Dong Chen, Norman Christ, Mike Clark, Saul Cohen, Calin Cristian, Zhihua Dong, Alan Gara, Andrew Jackson, Balint Joo, Chulwoo Jung, Richard Kenway, Changhoan Kim, Ludmila Levkova, Huey-Wen Lin, Xiaodong Liao, Guofeng Liu, Robert Mawhinney, Shigemi Ohta, Tilo Wettig, and Azusa Yamaguchi for the development of the QCDOC machine and its software. This development and the resulting computer equipment were funded by the U.S. DOE grant DE-FG02-92ER40699, PPARC JIF grant PPA/J/S/1998/00756 and by RIKEN. This work was supported by DOE grant DE-FG02-92ER40699 and we thank RIKEN, BNL and the U.S. DOE for providing the facilities essential for the completion of this work. C.J. is supported by SciDAC project of the U.S. Department of Energy, under the contract DE-AC02-98CH10886. 